\title{
Epidemiología del trauma maxilofacial, tratado quirúrgicamente en el Hospital de Urgencia Asistencia Pública: 3 años de revisión
}

\author{
Javier Cuéllar G. ${ }^{1}$, María Carla Prats P. ${ }^{1}$, Daniel Reyes C. ${ }^{1,2}$ y Víctor Sanhueza O. ${ }^{1}$
}

${ }^{1}$ Hospital de Urgencia Asistencia Pública, Dr. Alejandro del Río ${ }^{2}$ Departamento de Cirugía y Traumatología Bucal y Maxilofacial, Universidad de Chile. Santiago, Chile.

Recibido el 27 de noviembre de 2018 y aceptado para publicación el 12 de marzo de 2019.

Correspondencia a:

Dr. Javier Cuéllar G. javier.cuellar.gutierrez@gmail.
Epidemiology of maxillofacial trauma, surgically treated in the Emergency Hospital Public Assistance: 3 years of revision

Introduction: Maxillofacial trauma (MFT) is a relevant problem in highly complex hospital centers. Aim: To show and update the epidemiology of the maxillofacial trauma of surgical resolution under general anesthesia, in a major operating room, treated in the Emergency Hospital Public Assistance during the period from December 2014 to December 2017 and to compare it with the epidemiological reality of other high complexity hospital centers in Chile and abroad. Materials and Method: A retrospective study was carried out and the surgical protocols of the patients operated for maxillofacial trauma, in a major operating room, under general anesthesia, in a period of 37 months, were analyzed. The variables considered were diagnosis, age, gender, facial third and affected anatomic area. Results: 127 surgical procedures were performed, for the resolution of 259 fractures. The average age of the operated patients was 33 years, with predominance of the male gender $(85.8 \%)$, the lower third was the most affected $(77.2 \%)$, specifically in the parasymphysis area (22.4\%). Discussion: The exposed results agree with studies carried out internationally, but it has discordance in relation to the most affected anatomical area at national level comparing it with the other hospitals that analyzed its epidemiology. Conclusion: It is necessary to continue the epidemiological investigation of these pathologies and to unify diagnostic and treatment criteria in order to establish efficient prevention and treatment policies in relation to each service and jointly at the level of public health.

Key words: epidemiology; maxillofacial surgery; maxillofacial trauma.

\section{Resumen}

Introducción: El trauma maxilofacial (TMF) constituye un problema de relevancia en los centros hospitalarios de alta complejidad. Objetivo: Evidenciar y actualizar la epidemiología del trauma maxilofacial de resolución quirúrgica bajo anestesia general, en pabellón central tratado en el Hospital de Urgencia Asistencia Pública durante el período comprendido entre diciembre de 2014 a diciembre de 2017 y compararla con la realidad epidemiológica de otros centros hospitalarios de alta complejidad en Chile y en el extranjero. Materiales y Método: Se realizó un estudio retrospectivo en el que se analizaron los protocolos operatorios de los pacientes intervenidos quirúrgicamente por trauma maxilofacial, en pabellón central, bajo anestesia general, en un período de 37 meses. Las variables analizadas fueron diagnóstico, edad, género, tercio facial y zona anatómica afectada. Resultados: Se realizaron 127 procedimientos quirúrgicos, para resolver 259 fracturas. La edad promedio de los pacientes intervenidos fue de 33 años, con predominio del género masculino $(85,8 \%)$; el tercio inferior fue el más afectado $(77,2 \%)$, específicamente en la zona parasinfisiaria mandibular (22,4\%). Discusión: Los resultados expuestos concuerdan con estudios realizados internacionalmente, pero tiene discordancia en relación a la zona anatómica más afectada al comparalo con los otros centros que analizaron su epidemiología. Conclusión: Resulta necesario continuar la investigación epidemiológica de estas patologías y unificar criterios de diagnóstico y tratamiento para poder establecer políticas de prevención y tratamiento eficientes en relación a cada servicio y en conjunto a nivel de salud pública.

Palabras clave: epidemiología; cirugía maxilofacial; trauma maxilofacial. 


\section{Introducción}

El trauma maxilofacial (TMF) constituye un problema de relevancia en los centros hospitalarios de alta complejidad. Las consecuencias funcionales y estéticas generadas por éste necesitan de un diagnóstico asertivo y eficiente para lograr decisiones terapéuticas oportunas, evitando futuras complicaciones, tales como recidivas y/o secuelas.

Este tipo de trauma, frecuentemente se asocia a una severa morbilidad, pérdida de función, desfiguramiento (Figura 1) y un costo económico significativo. La gran mayoría de estos casos requieren de hospitalización y resolución quirúrgica bajo anestesia general en pabellón central.

Los factores que determinan el tratamiento del TMF son principalmente: la edad del paciente, tipo de fractura, ubicación anatómica y morbilidad asociada, entre otras ${ }^{1,2}$

Actualmente, el Hospital de Urgencia Asistencia Pública Dr. Alejandro del Río (HUAP), de Santiago de Chile, es un hospital público, asistencial-docente, enfocado al tratamiento de urgencias y emergencias de alta complejidad en adultos, que recibe y resuelve patología aguda de TMF.

El objetivo del presente artículo es evidenciar y actualizar la epidemiología del TMF de resolución quirúrgica bajo anestesia general, en pabellón central tratado en el HUAP durante el período comprendido entre diciembre de 2014 a diciembre de 2017 y compararla con la realidad epidemiológica de otros centros hospitalarios de alta complejidad en Chile y en el extranjero.

\section{Materiales y Método}

Se realizó un estudio observacional retrospectivo, mediante la recolección y revisión de los protocolos quirúrgicos de las cirugías realizadas en pabellón central, bajo anestesia general. Dichas cirugías fueron realizadas por especialistas en Cirugía y Traumatología Bucal y Maxilofacial, integrando equipos multidisciplinarios para la resolución quirúrgica de los casos que lo ameriten.

Se incluyeron todos los pacientes con patología aguda de origen traumática del territorio maxilofacial, de ambos géneros, tratados quirúrgicamente y en pabellón central en el HUAP, en un período de tres años comprendido entre diciembre de 2014 y diciembre de 2017.

Fueron excluidos todos los casos clínicos de trauma maxilofacial ambulatorio. Por otra parte, las fracturas nasales puras o de los huesos propios de la nariz fueron excluidas del estudio.

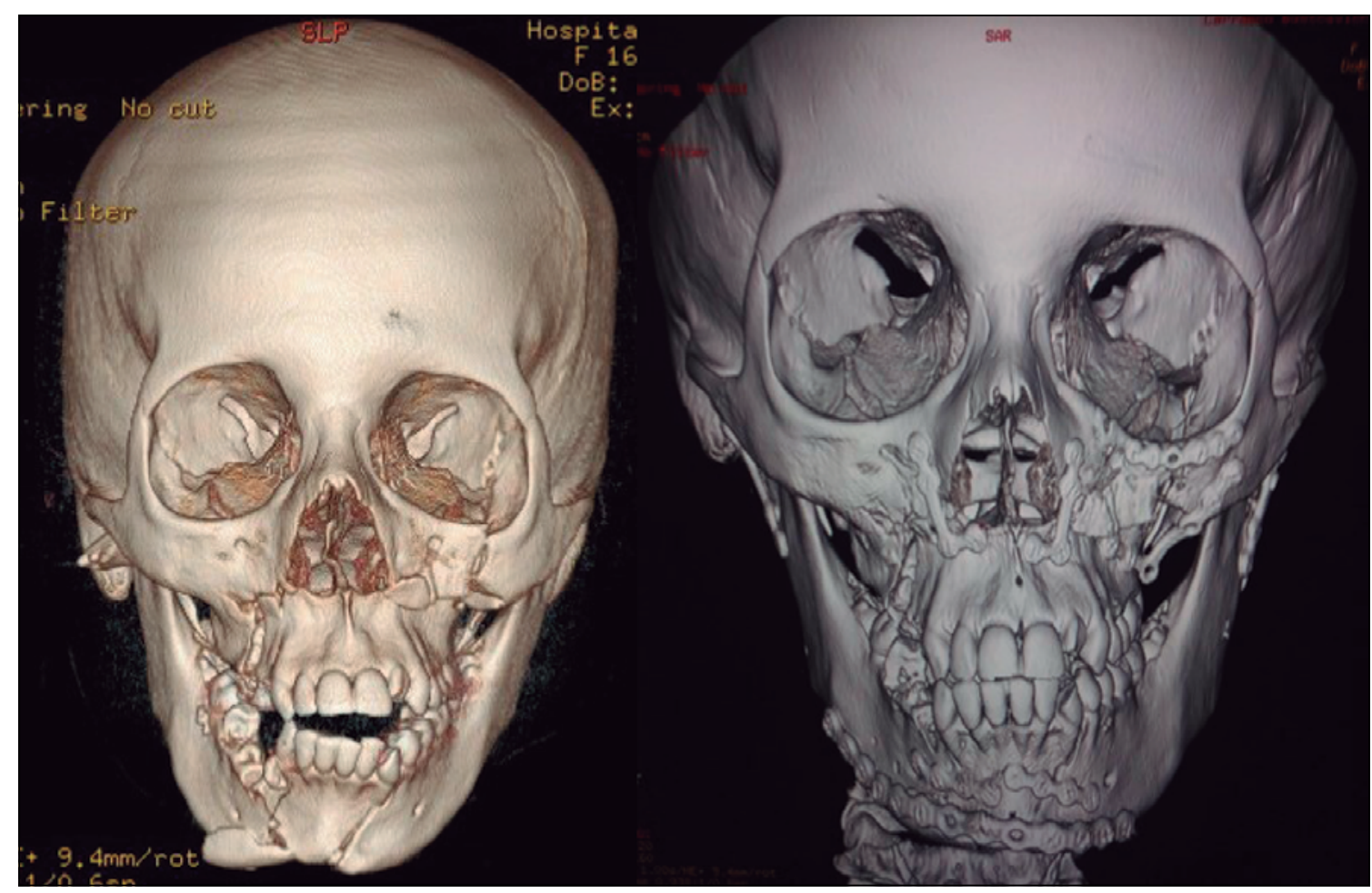

Figura 1. Caso de trauma maxilofacial tratado en base a reducción abierta y fijación interna rígida en HUAP. 
Tabla 1. Distribución de pacientes según edad

\begin{tabular}{|lcc|}
\hline & $\begin{array}{r}\text { Edad } \\
\mathbf{n}\end{array}$ & $\mathbf{\%}$ \\
\hline $15-19$ & 15 & $11,8 \%$ \\
\hline $20-29$ & 43 & $33,9 \%$ \\
\hline $30-39$ & 28 & $22,0 \%$ \\
\hline $40-49$ & 22 & $17,3 \%$ \\
\hline $50-59$ & 14 & $11,0 \%$ \\
\hline $60-$ más & 5 & $3,9 \%$ \\
\hline Total & 127 & 100 \\
\hline
\end{tabular}

Tabla 2. Distribución de frecuencia de rasgos de fracturas según tercio y zona anatómica afectada

\begin{tabular}{|c|c|c|c|c|}
\hline \multirow{2}{*}{$\begin{array}{l}\text { Localización } \\
\text { facial }\end{array}$} & \multirow{2}{*}{$\begin{array}{l}\text { Ubicación anatómica } \\
\text { fractura }\end{array}$} & \multicolumn{2}{|c|}{ n de fracturas } & \multirow{2}{*}{$\begin{array}{c}\% \\
\text { del total }\end{array}$} \\
\hline & & & $\begin{array}{c}\% \\
\text { por tercio }\end{array}$ & \\
\hline \multirow[t]{3}{*}{ Tercio superior } & Frontal & 1 & $100 \%$ & $0,4 \%$ \\
\hline & Pared anterior seno frontal & 0 & $0 \%$ & $0,0 \%$ \\
\hline & Subtotal & 1 & 100 & 0,4 \\
\hline \multirow[t]{10}{*}{ Tercio medio } & Cuerpo cigomático & 27 & $48,2 \%$ & $10,4 \%$ \\
\hline & Arco cigomático & 4 & $7,1 \%$ & $1,5 \%$ \\
\hline & Maxilar & 13 & $23,2 \%$ & $5,0 \%$ \\
\hline & Lefort I & 4 & $7,1 \%$ & $1,5 \%$ \\
\hline & Lefort II & 2 & $3,6 \%$ & $0,8 \%$ \\
\hline & Lefort III & 0 & $0,0 \%$ & $0,0 \%$ \\
\hline & Alveolar & 2 & $3,6 \%$ & $0,8 \%$ \\
\hline & Piso de órbita & 4 & $7,1 \%$ & $1,5 \%$ \\
\hline & Naso-órbito-etmoidal & 0 & 0 & $0,0 \%$ \\
\hline & Subtotal & 56 & $100 \%$ & $21,5 \%$ \\
\hline \multirow[t]{9}{*}{ Tercio inferior } & Sínfisis & 7 & $3,50 \%$ & $2,7 \%$ \\
\hline & Parasínfisis & 58 & $29,00 \%$ & $22,4 \%$ \\
\hline & Cuerpo & 39 & $19,50 \%$ & $15,1 \%$ \\
\hline & Ángulo & 47 & $23,50 \%$ & $18,1 \%$ \\
\hline & Rama & 6 & $3,00 \%$ & $2,3 \%$ \\
\hline & Coronides & 0 & $0,00 \%$ & $0,0 \%$ \\
\hline & Cóndilo & 41 & $20,50 \%$ & $15,8 \%$ \\
\hline & Alveolar & 2 & $1,00 \%$ & $0,8 \%$ \\
\hline & Subtotal & 200 & 100 & 77,2 \\
\hline \multirow[t]{3}{*}{ Panfacial } & Subtotal & 2 & 100 & 1 \\
\hline & & 2 & 100 & 0,8 \\
\hline & Total & 259 & & 100 \\
\hline
\end{tabular}

La recolección de los datos obtenidos fue organizada y analizada en variables de frecuencia, zona anatómica de la fractura maxilofacial, edad y género.

En relación a la edad, los datos fueron distribuidos en rangos comprendidos entre: los 15 y 19 años, 20 y 29 años, 30 y 39 años, 40 y 49 años, 50 y 59 años y el grupo de 60 años o más (Tabla 1).

Las zonas anatómicas afectadas por el TMF fueron divididas según el tercio facial que fue afectado y éste a su vez se subdividió en el tipo de fractura y/o combinación de huesos involucrados: tercio superior (hueso frontal y pared anterior del seno frontal), tercio medio (cuerpo cigomático, arco cigomático, hueso maxilar, fracturas tipo Lefort I, Lefort II, Lefort III, reborde alveolar, piso de órbita y complejo naso-órbito-etmoidal), tercio inferior (sínfisis, parasínfisis, cuerpo mandibular, ángulo mandibular, rama mandibular, coronoides , cóndilo y reborde alveolar). Además, fueron consideradas las fracturas panfaciales como un sólo tipo para efectos estadísticos (Tabla 2).

Para verificar si existe una asociación entre la variable estudiada y el mes del año en que ocurre el trauma, se utilizó el test estadístico de MannKendall. Existiendo una significancia estadística con $p \leq 0,05$. Las variables a estudiar consideradas en este estudio fueron la frecuencia del TMF en el total de pacientes y frecuencia del TMF total por género relacionado al mes del año. Para analizar por mes en el test estadístico se realizó la sumatoria total de pacientes operados en cada mes, durante los 3 años.

\section{Resultados}

El total de procedimientos quirúrgicos que requirieron ser realizados en pabellón central bajo anestesia general durante un período de 37 meses fue 127 , el promedio correspondió a 3,4 procedimientos por mes. Se realizaron 109 procedimientos quirúrgicos correspondientes a traumatismos intervenidos en hombres y 18 procedimientos quirúrgicos a traumatismos intervenidos en mujeres, cuya relación estimada fue de 6:1

Los grupos etarios se describen de la siguiente manera: el $11,8 \%$ de los procedimientos fueron en el grupo de 15 a 19 años, el 33,9\% en el grupo de 20 a 29 años, $22 \%$ en el de 30 a 39 años, $17,3 \%$ en el de 40 a 49 años, $11 \%$ en el de 50 a 59 años y por último 3,9\% entre los 60 y más años (Tabla 1).

La edad promedio del total de pacientes fue de 33 años, el paciente más joven fue de 15 años y el más longevo fue de 92 años. El rango de edad más 
afectado fue el comprendido entre los 20 y los 29 años con un porcentaje de $33,9 \%(n=43)$, seguido por el grupo comprendido entre los 30 y los 39 años con un $22 \%(\mathrm{n}=28)$. Estos 2 grupos etarios representaron más de la mitad de los pacientes afectados (Tabla 1).

Del total de procedimientos quirúrgicos asociados a trauma maxilofacial resueltos bajo anestesia general en HUAP $(n=127)$, se trataron 259 fracturas. La distribución de las fracturas fue en relación a la ubicación anatómica de la fractura, específicamente dividido por los tercios de la cara. El tercio superior presentó 1 fractura $=0,4 \%$, el tercio medio 56 fracturas $=21,6 \%$ y el tercio inferior, siendo éste el más prevalente, presentó 200 fracturas $=77,2 \%$ (Tabla 2).

Dentro del tercio superior, éste presentó 2 fracturas en el hueso frontal.

En el tercio medio, se registraron: 27 fracturas en el cuerpo cigomático, 4 fracturas en el arco cigomático, 13 fracturas en el hueso maxilar, 4 fracturas tipo Le Fort I, 2 fracturas tipo Le Fort II, no se reportaron fracturas tipo Le Fort III, 2 fracturas en el hueso alveolar, 4 fracturas en el piso de órbita.

El tercio inferior fue el más afectado en comparación a los otros dos tercios, 200 fracturas que equivalen a $77,2 \%$, de las cuales 7 fracturas correspondieron a sinfisiarias, 58 fracturas fueron parasinfisiarias, 39 fracturas de cuerpo mandibular, 47 fracturas de ángulo mandibular, 7 fracturas de la rama mandibular, 41 fracturas de cóndilo mandibular, 2 fracturas del hueso alveolar inferior y no se presentaron fracturas de la apófisis coronoides (Tabla 2).

El test de Mann-Kendall fue aplicado al trauma maxilofacial del total de pacientes en relación a cada mes del año. Adicionalmente, el test estadístico, se aplicó al TMF total en hombres y del mismo modo al total en mujeres en relación a cada mes del año. Los resultados entre el total de pacientes y cada mes del año no fueron significativos, $p=0,061$, sin embargo, la relación entre el total de TMF en hombres y cada mes del año muestra un resultado estadísticamente significativo, $p=0,019$. Existiendo un alza abrupta en la incidencia del TMF entre los meses de octubre y diciembre en los tres años (Figuras 2 y 3 respectivamente). La asociación entre TMF de mujeres y época del año mostró un resultado no significativo con un $\mathrm{p}=0,931$ (Figura 4).

\section{Discusión}

El TMF ha ido en aumento en los últimos tres años en la población revisada en el presente estudio,

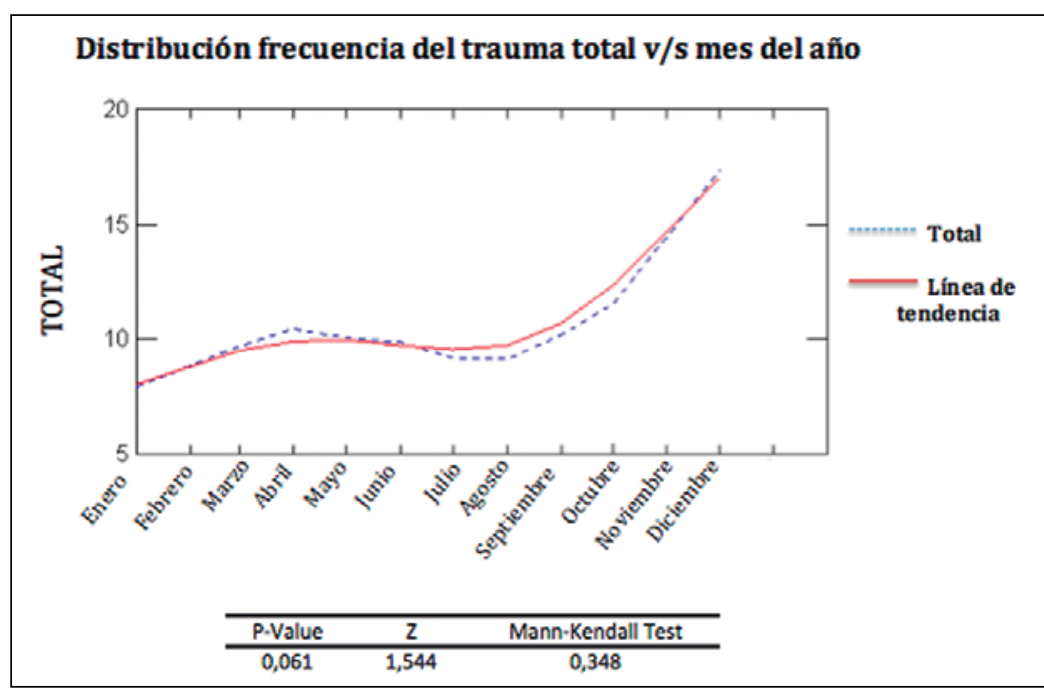

Figura 2. Relación entre frecuencia del trauma total y mes del año.

Distribución frecuencia del trauma en hombres $\mathrm{v} / \mathrm{s}$ mes del año

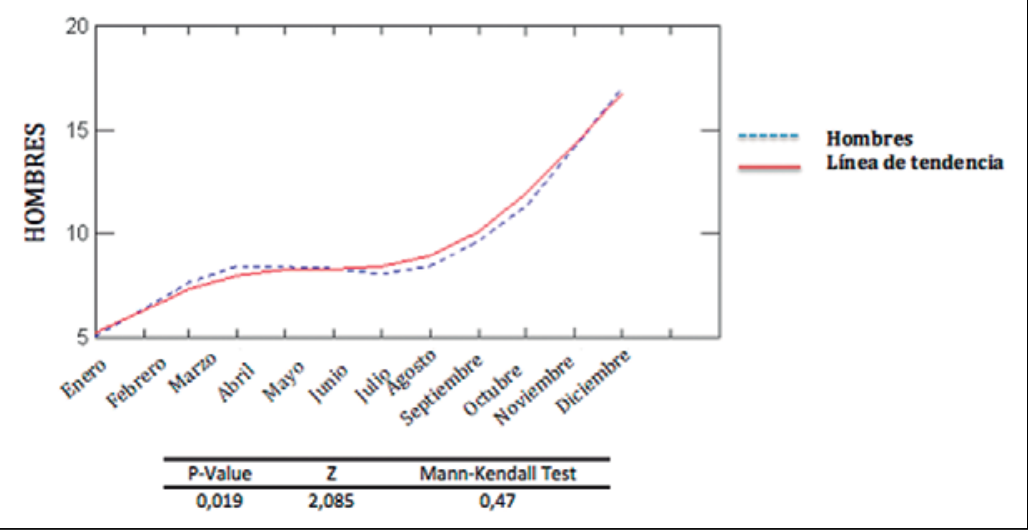

Figura 3. Relación entre frecuencia del trauma total de hombres y mes del año.

Distribución frecuencia del trauma en mujeresv/s mes del año

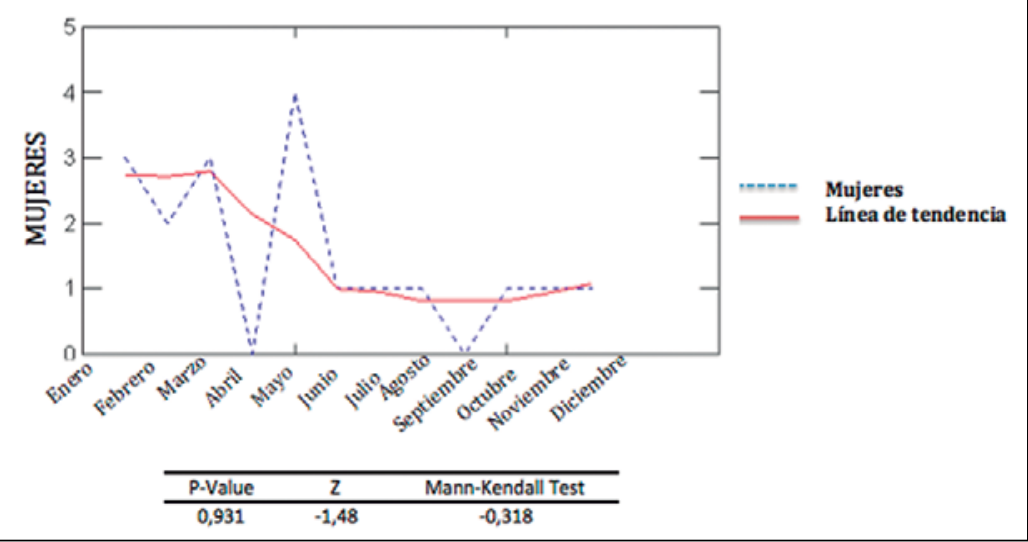

Figura 4. Relación entre frecuencia del trauma total de mujeres y mes del año. 
principalmente en hombres, específicamente en la edad comprendida entre los 20 y 29 años. Considerando la importancia funcional, estética y de salud para el paciente, adicionalmente también por el aporte estadístico informativo al centro hospitalario, que conlleva su resolución quirúrgica, se hace importante y necesario mantener una actualización constante de este análisis epidemiológico. Para así poder estimar la incidencia, prevalencia y mortalidad, más sus tendencias; poder evaluar estrategias de diagnósticos asertivos; estimar y modelar la calidad de vida por tratamiento de trauma, entre otros. A su vez, generar un registro hospitalario que provea información de gestión administrativa de los tipos de trauma maxilofacial; estimar la distribución $\mathrm{y}$ frecuencia de los mismos y de tal manera poder mejorar la asignación de recursos; monitorear los patrones de tratamiento y de cuidado ya sea con tratamientos convencionales de cada centro o dentro de un protocolo de investigación clínico.

En relación al TMF y su distribución por género, $85,8 \%$ fueron hombres y 14,2\% mujeres. Esta prevalencia masculina coincide con estudios internacionales realizados en Brasil (Brasileiro) ${ }^{3}$, Grecia

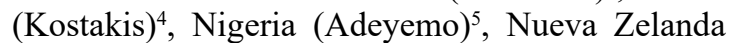
(Lee) ${ }^{6}$.

En el ámbito nacional, los centros hospitalarios nacionales de forma individual han reportado su epidemiología del trauma maxilofacial en base a fichas clínicas, protocolos operatorios, entre otros, encontrando en ellos resultados diferentes a los expuestos en el presente estudio ${ }^{7-17}$. Principalmente, esta diferencia se asocia a variantes en la zona geográfica, densidad poblacional, estatus socioeconómico, estilos de vida, etc. Adicionalmente en la literatura se han descrito reportes que están enfocados a TMF generalizado, no pudiendo diferenciar entre pacientes tratados bajo anestesia general en pabellón central, pabellón ambulatorio o de resolución no quirúrgica

Servicios de cirugía maxilofacial de centros de alta complejidad, realizaron estudios observacionales descriptivos a partir de su estadística. Éstos mostraron una amplia diferencia en relación a las variables de sitio anatómico de la fractura, pero mantienen similitud en relación a la frecuencia entre casos analizados por género, inclinándose por el género masculino como el predominante y también en relación al rango de edad más afectado, que corresponde al rango de 20-29 años.

Cabe mencionar que los estudios chilenos realizados por Raposo ${ }^{7}$ y Pérez ${ }^{8}$ están hechos en base a los protocolos operatorios efectuados en pabellón central bajo anestesia general, lo que coincide con la metodología de nuestro estudio y permite realizar asociaciones más fidedignas entre los resultados.

Los estudios realizados por Faille ${ }^{9}$ en el hospital Carlos Van Buren de Valparaíso, Zapata ${ }^{10}$ y Pache$\mathrm{co}^{11}$ en el Instituto Traumatológico de Santiago de Chile, González ${ }^{12}$ en el Hospital Clínico Mutual de Seguridad C.Ch.C. de Santiago de Chile, Medina ${ }^{13}$ en el Hospital Regional Hernán Henríquez Aravena de Temuco y Zúñiga ${ }^{14}$ en el Hospital Barros Luco Trudeau de Santiago de Chile fueron realizados en base a fichas clínicas. Mientras que el estudio realizado por Venegas ${ }^{15}$ en el Hospital de La Serena, fue en base a la epicrisis. El estudio realizado por Iribarren ${ }^{16}$ en el Hospital de Coquimbo no especifica su método para recolección de datos.

Los resultados presentados en los estudios en base a fichas clínicas no discriminan si éstos fueron tratados bajo anestesia general, ambulatorios o de resolución no quirúrgica. Por lo que sólo permite realizar una comparación general con nuestro estudio, a excepción de Faille ${ }^{9}$ que basó sus resultados en base a procedimientos realizados en pabellón central obteniendo características de la ficha clínica.

De esta forma los estudios realizados en Chile muestran una amplia diferencia en la frecuencia entre hombres y mujeres, coincidiendo con los resultados presentados durante tres años en el HUAP. Principalmente Faille ${ }^{9}$, pero más aún en los estudios de Raposo $^{7}$ y de Pérez ${ }^{8}$ que realizaron su base de datos en base a protocolos operatorios bajo anestesia general.

Según el tipo de fractura facial nuestro estudio reportó que la fractura mandibular con un $77,2 \%$ fue la más prevalente, en donde la más frecuente fue la zona parasinfisiaria con $22,4 \%$, seguida por la fractura de ángulo mandibular con un 18,1\%.

Resultados similares fueron encontrados por Kostakis $^{4}$ en Grecia (56\%) y por Brasileiro ${ }^{3}$ en Brasil (44,2\%), en cuanto a que la mayoría fueron fracturas mandibulares. Al compararlo con la realidad de nuestro país, Raposo ${ }^{7}$ y encontro un 70,4\% de fracturas mandibulares, pero no especificaron zona anatómica. Pérez ${ }^{8}$ también reportó una mayoría en las fracturas mandibulares con un $48,8 \%$, en donde el sitio anatómico más afectado fue el ángulo mandibular. En el estudio realizado por Faille ${ }^{9}$ un $47,8 \%$ fueron fracturas mandibulares, siendo las más comunes la de cóndilo, seguidas de las fracturas parasinfisiaria y de ángulo mandibular.

A diferencia del estudio realizado por Medina ${ }^{13}$ que determinó que la fractura más prevalente resultó ser el complejo NOE (Naso-Orbito-Etmoidal) con un $55,6 \%$, seguido de las fracturas dentoalveolares $22,2 \%$ y mandibular $13,3 \%$. Estas diferencias se pueden explicar porque incluyeron todas las consul- 
tas de urgencia, mientras que en el presente estudio sólo abarcó los casos de resolución quirúrgica en pabellón central.

En referencia a los estudios de Zapata ${ }^{10}$ y Pache$\mathrm{co}^{11}$ en el Instituto Traumatológico de Santiago de Chile, los resultados se pueden comparar sólo por zona anatómica, debido que estos estudios fueron específicos para tercio inferior y tercio medio respectivamente. Zapata ${ }^{10}$ determinó a la fractura de ángulo mandibular como la más prevalente con 42,8\% mientras que Pacheco ${ }^{6}$ reportó a las fracturas de huesos nasales como las más prevalentes $(44,71 \%)$, seguidas por las fracturas del complejo cigomático $(44,57 \%)$.

En el estudio de González ${ }^{12}$ el complejo cigomático fue la zona anatómica de fractura más prevalente con un $48 \%$. Venegas ${ }^{15}$, en cambió reporta a la fractura dentoalveolar como la más frecuente, sin discriminar en superior e inferior con un 43,3\%, Iribarren et al. $(2002)^{16}$; por otra parte reportó a las fracturas fronto orbitaria y cigomático malar, ambas con un $19 \%$ y $14 \%$, como las de mayor prevalencia.

En el presente estudio la fractura de ángulo mandibular se presenta como la segunda fractura más prevalente con un $18,1 \%$. Mientras que las fracturas del complejo cigomático correspondieron a un $11,9 \%$.

Por otra parte, es importante destacar que efectivamente existe una asociación estadísticamente significativa entre el trauma en hombres tratados bajo anestesia general en pabellón central en el HUAP y la época del año, cuyo resultado en el test de Mann Kendall fue de $p=0,019$. Lo que nos indica que entre los meses de octubre y diciembre lo más probable es que aumente significativamente el trauma maxilofacial en pacientes hombres.

Cuando analizamos el trauma en mujeres, en el test de Mann Kendall el resultado fue de $\mathrm{p}=0,931$. Lo que indica que, en este estudio, no se puede asociar el trauma maxilofacial en mujeres tratadas en el HUAP y la época del año. Probablemente este resultado se deba al reducido tamaño de la muestra en pacientes de género femenino.

Por último, el trauma maxilofacial en el total de pacientes tratados bajo anestesia general en pabellón central del HUAP arroja un resultado relevante e interesante en el test de Mann Kendall, p $=0,061$. Si bien es un resultado no estadísticamente significativo, sí presenta una relevancia estadística, debido a que si se mantiene este estudio del trauma maxilofacial del HUAP en los próximos años, aumentará el tamaño de la muestra y de la población en estudio, por lo que podemos inferir que sí habrá una significancia estadística y una real asociación entre el trauma maxilofacial total de pacientes y la época del año.

Debido a que el presente estudio se basa sólo en protocolos operatorios quirúrgicos de pabellón central bajo anestesia general, es imposible registrar la causa del trauma. Cabe destacar que el protocolo operatorio quirúrgico es un documento que realiza el cirujano sobre la intervención quirúrgica en donde se puede identificar el diagnóstico preintervención, sin necesidad de tener que acudir a la ficha clínica. Sería de un gran aporte poder acudir a los datos de ella, aunque es necesario el consentimiento del paciente y la aprobación del comité de ética correspondiente, para así obtener datos principalmente de la etiología, anamnesis y tiempo de hospitalización para poder realizar un análisis más exhaustivo.

\section{Conclusión}

El trauma maxilofacial tratado en el Hospital de Urgencia Asistencia Pública se relaciona mayoritariamente en hombres de un rango de 20 a 29 años, demostrando asociación de casos nuevos de trauma maxilofacial en el año entre los meses de octubre y diciembre. Los resultados de este estudio coinciden principalmente con la literatura nacional en relación a edad, género y distribución anatómica de la fractura.

El estudio estadístico extendido y a largo plazo del trauma maxilofacial es necesario ya que proporciona información fundamental para el análisis epidemiológico. Permitiendo estimar resultados y generar información que permita gestión administrativa a nivel del centro hospitalario.

Sería interesante contar con estudios multicéntricos que permitan aumentar el número de casos para así generar una muestra más representativa y a su vez obtener resultados más fidedignos; considerando además la unificación de bases de datos de los distintos centros hospitalarios que permitiría una mayor transparencia epidemiológica en beneficio de la comunidad.

\section{Responsabilidades éticas}

Protección de personas y animales. Los autores declaran que para esta investigación no se han realizado experimentos en seres humanos ni en animales.

Confidencialidad de los datos. Los autores declaran que en este artículo no aparecen datos de pacientes.

Conflictos de interés: no hay. 


\section{Bibliografía}

1. Hupp J, Ellis E, Tucker M. Contemporary Oral and Maxillofacial Surgery. Ochs M, Tucker M (Editors). Missouri: Elsevier; 2014. 491-518. (Mark W, Ochs R, Myron R. Management of Facial Trauma; vol 6).

2. Curtis W, Horswell B. Panfacial Fractures: an approach to management. Oral Maxillofac Surg Clin North Am. 2013;25:649-60.

3. Brasileiro BF, Passeri LA. Epidemiological analysis of maxillofacial fractures in Brazil: a 5-year prospective study. Oral Surg Oral Med Oral Pathol Oral Radiol Endod. 2005;102:28-34.

4. Kostakis G, Stathopoulos P, Dais P, Gkinis G, Igoumenakis D, Mezitis M, et al. An epidemiologic analysis of 1,142 maxillofacial fractures and concomitant injuries. Oral Surg Oral Med Oral Pathol Oral Radiol Endod. 2012;114:69-73.

5. Adeyemo W, Ladeinde A, Ogunlewe $\mathrm{O}$, James $\mathrm{O}$. Trends and characteristics of oral and maxillofacial injuries in Nigeria: a review of the literature. Head Face Med. 2005;4:1-7.

6. Lee K. Global Trends in Maxillofacial Fractures. Cranial Maxillofac Trauma Reconstruction 2012;5:213-22.
7. Raposo A, Preisler G, Salinas F, Muñoz C, Monsalves MJ. Epidemiología de las fracturas maxilofaciales tratadas quirúrgicamente en Valdivia, Chile: 5 años de revisión. Rev Esp Cirug Oral y Maxilofac. 2013;35:18-22.

8. Faille A, Badillo O. Caracterización de los casos de fracturas maxilofaciales operados en el Hospital Carlos Van Buren, Chile, entre los años 20102014. Rev Esp Cirug Oral y Maxilofac. 2018;40:169-75.

9. Pérez H, Donoso T, Mardones M, Bravo R. Epidemiología de Tratamientos Quirúrgicos Maxilofaciales en un Hospital Público en Santiago de Chile: Estudio Retrospectivo de 5 Años. Int. J. Odontostomat. 2015;9:37-41.

10. Zapata S, Pacheco C, Núñez C, Gazitúa G, Cerda P. Epidemiología de las fracturas mandibulares tratadas quirúrgicamente en el Instituto Traumatológico de Santiago (Chile): 10 años de revisión. Rev Esp Cir Oral Maxilofac. 2015;37:123-70.

11. Pacheco C, Zapata S. Fracturas del tercio medio facial operadas en el Instituto Traumatológico de Santiago (Chile): 10 años de revisión. J Oral Res. 2012;1:71-6.

12. González E, Pedemonte C, Vargas I, Lazo D, Pérez H, Canales M, et al. Facial fractures in a reference center for Level I Traumas. Descriptive study. Rev Esp Cirug Oral y Maxilofac. 2015;37:65-70.

13. Medina MJ, Molina P, Bobadilla L, Zaror R, Olate S. Fracturas Maxilofaciales en Individuos Chilenos. Int. J. Morphol. 2006;24:423-8.

14. Zúñiga E, Yoma T, Núñez C, Quezada G, Sung, H. Epidemiological profile of craniofacial trauma in the Hospital Barros Luco Trudeau, Santiago, Chile. Period 2006-2010. Int J Oral Maxillo Surg. 2011;40:1112.

15. Venegas O, Nicola M, Barrera R, Zambra M, Olivos B, Tovar R. Estudio descriptivo del traumatismo máxilofacial en el Hospital de La Serena entre los años 2004-2011. Rev Chil Cir. 2013;65:525-9.

16. Iribarren $\mathrm{O}$, Carvajal M. Complicaciones de la fijación interna con placas de Titanio de las fracturas Maxilofaciales. Rev Chil Cir. 2002;54:368-72.

17. Sandoval M, Reyes D, Sanhueza V. Epidemiología de la patología quirúrgica que afecta al territorio maxilofacial, tratada bajo anestesia general en el Hospital de Urgencia Asistencia Pública entre 2014 y 2016. Rev Chil Cir. 2017;69:289-96. 